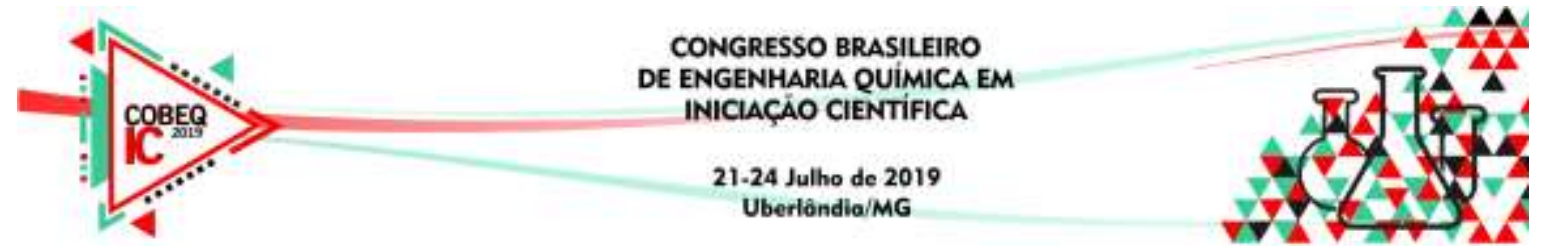

\title{
CALOR ESPECÍFICO DE POLPAS DE FRUTAS DA REGIÃO NORTE DO BRASIL ESTUDO DE CASO: AÇAÍ (Euterpe oleracea, Mart.).
}

\author{
A. C. P. DIAS ${ }^{1}$, M. D. O. das CHAGAS ${ }^{2}$, F. de S. FERRAZ ${ }^{3}$, M. \\ M. A. de JESUS ${ }^{4}$ e L. M. GRAJALES ${ }^{1,5}$ \\ ${ }^{1}$ Universidade Federal do Tocantins, Curso de Engenharia de Alimentos \\ ${ }^{2}$ Universidade Federal do Tocantins, Curso de Engenharia Elétrica \\ ${ }^{3}$ Universidade Federal do Tocantins, Programa de Pós-Graduação em Tecnologia e Ciência \\ de Alimentos \\ ${ }^{4}$ Universidade Tecnológica Federal do Paraná, Departamento de Física \\ ${ }^{5}$ Universidade Federal do Tocantins, Programa de Pós-Graduação em Agroenergia \\ E-mail para contato: ana.dias@uft.edu.br
}

\begin{abstract}
RESUMO - O açaí (Euterpe oleracea Mart.) é um fruto exótico e um alimento básico na região norte do Brasil, é consumido tradicionalmente in natura ou na forma de sorvetes, geleias, doces e sucos. Para aumentar sua vida útil, diversos processos térmicos como congelamento, criocongelamento, concentração por evaporação, branqueamento, pasteurização, esterilização, secagem e desidratação podem ser realizados. Portanto, o conhecimento das propriedades térmicas para o seu processamento tem grande importância, especificamente o calor específico $(\mathrm{Cp})$ para o projeto de equipamentos e simulação de processos. Assim, este trabalho tem como objetivo determinar experimentalmente o $C p$ da polpa de açaí. Para isto, um calorímetro adiabático foi construído com uma garrafa térmica segundo o método descrito por Ferraz et al. (2018). O princípio de funcionamento está baseado no efeito Joule, no qual a energia elétrica é convertida em energia térmica. As leituras das temperaturas foram registradas utilizando a plataforma Arduino. Os frutos obtidos em feiras de Palmas-To foram branqueados, despolpados e congelados, a polpa foi utilizada na determinação do $C p$. Através de um balanço de energia do sistema, o Cp da polpa de açaí na faixa de temperatura de 8 a $45^{\circ} \mathrm{C}$ foi determinado. $\mathrm{O}$ valor do calor especifico encontrado para o açaí foi de $0,4512 \mathrm{cal} / \mathrm{g}^{\circ} \mathrm{C}$. $\mathrm{O}$ valor de $C p$ encontrado para o açaí se aproxima de dados encontrados na literatura para outras frutas de composição similar.
\end{abstract}

\section{INTRODUÇÃO}

O açaizeiro (Euterpe oleracea Mart.), de origem nativa da América Central e do Sul, é considerado como a palmeira mais produtiva da região amazônica. Destaca-se por ser um fruto exótico e é consumido como um alimento básico nessa região do Brasil, além de ser considerada de grande importância econômica, cultural e social do Norte do país. O açaizeiro frutifica na estação seca entre julho e dezembro, seus frutos são arredondados com diâmetro de $1-1,5 \mathrm{~cm}$, com coloração roxa-escura quando maduros. Possuem um mesocarpo polposo de $1 \mathrm{~mm}$ de espessura e um endocarpo fibroso, a parte comestível do fruto representa em média $26,54 \%$ da 


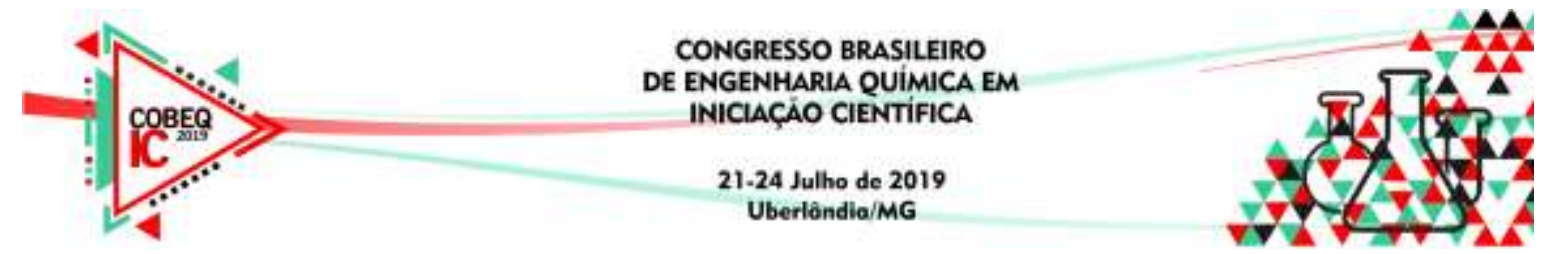

sua massa, enquanto a semente compõe a maior parte da fruta (CEDRIM, 2018; FREITAS et al., 2015; SOUZA, 2015).

O açaí é uma fruta altamente perecível devido às condições ambientais desfavoráveis, tais como, temperatura e umidade relativa elevadas, a falta de assepsia no manuseio favorece a proliferação de microrganismos e reações enzimáticas acelerando a deterioração da fruta. Sendo assim, o despolpamento deve ser realizado dentro de $24 \mathrm{~h}$ após a coleta e, mesmo se mantida refrigerada, possui uma vida útil máxima de $12 \mathrm{~h}$, sendo então necessário submetê-lo a processamentos térmicos (CEDRIM, 2018; SILVA et al., 2017; SOUZA, 2015).

Para o seu processamento é necessário o conhecimento das propriedades termofísicas, pois estas afetam a transferência de calor e de massa, consequentemente, o armazenamento e vida de prateleira. Estas propriedades são utilizadas no projeto, otimização e implementação de projetos na área de engenharia de alimentos, exigindo informações precisas das propriedades térmicas dos alimentos líquidos e semissólidos como densidade, condutividade térmica, difusividade térmica e calor específico em função da variação de temperatura (MOURA et al.,2016; SOUZA, 2015).

Levando em consideração a importância do conhecimento das propriedades térmicas para o processamento de alimentos, este trabalho tem como objetivo determinar experimentalmente o calor específico da polpa de açaí (Euterpe oleracea, Mart.) em uma faixa de temperatura de 8 a $45^{\circ} \mathrm{C}$.

\section{METODOLOGIA}

Para a obtenção da polpa de açaí os frutos foram adquiridos em feiras municipais de Palmas-TO, armazenados e transportados para o laboratório de Tecnologia de Frutas e Hortaliças da Universidade Federal do Tocantins (UFT), campus de Palmas. Os frutos foram lavados com água corrente para remoção das sujidades e branqueados a $80^{\circ} \mathrm{C}$ por 10 segundos, seguidos de resfriamento, de acordo com a metodologia descrita por Silva et al. (2017). Em seguida foi realizado o despolpamento, em despolpadeira bonina $0.25 \mathrm{DF}$, compacta com capacidade de processamento de $100 \mathrm{~kg} / \mathrm{h}$ e motor elétrico de $1,0 \mathrm{cv}$. Foi utilizado um litro de água potável para cada cinco quilogramas do fruto, em seguida a polpa foi embalada em sacos de polietileno de baixa densidade e congelada.

Análises físico-químicas foram realizadas para a matéria seca. A secagem foi realizada em estufa a $70^{\circ} \mathrm{C}$ até peso constante. Uma vez seca a amostra, esta foi triturada em processador Arno (Magiclean Duetto) até a obtenção de um pó. O açaí foi caracterizado com relação a: umidade, proteína, lipídeos, cinzas, carboidrato, $\mathrm{pH}$ e sólidos solúveis totais. As análises foram realizadas seguindo os métodos descritos pelo Instituto Adolfo Lutz (2008), para análise de alimentos.

O calor específico do açaí foi determinado experimentalmente através de uma adaptação do método descrito por Ferraz (2018). Um calorímetro adiabático foi construído em parceria com a Universidade Tecnológica Federal do Paraná -UTFPR, em Apucarana-PR, no laboratório de Pesquisa II. Este utiliza uma resistência elétrica que converte a energia em térmica. 


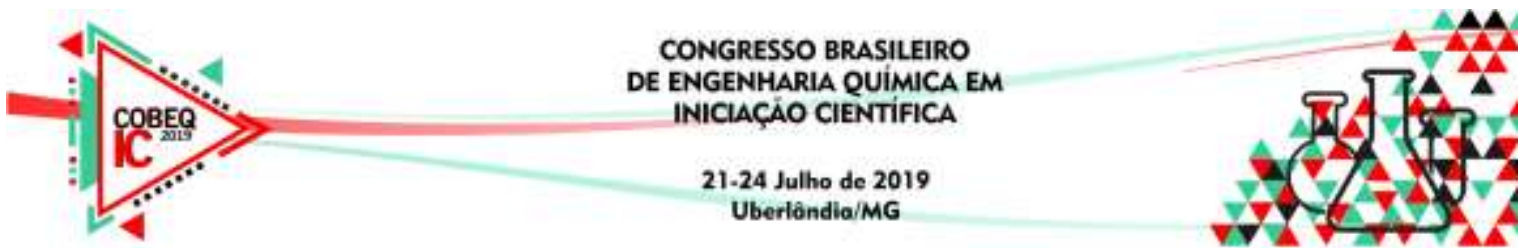

Pela lei de conservação da energia apresentada na Equação (1), o calor específico é calculado de acordo com a Equação (2).

$$
\begin{gathered}
Q_{\text {res }}=Q_{c a l}+Q_{a m .} \\
\frac{V^{2}}{R}\left(t_{2}-t_{1}\right)-C p_{c a l}\left(T_{2}-T_{1}\right)=C p_{a m .}\left(T_{2}-T_{1}\right)
\end{gathered}
$$

Onde: $Q_{\text {res }}=$ é o calor gerado pela corrente que percorre a resistência elétrica; $Q_{c a l}=$ calor absorvido pelo calorímetro (cal); Qam é o calor ganho pela amostra na faixa de temperatura; V = diferença de potencial elétrico, em volts $(\mathrm{V}) ; R=$ resistência elétrica do condutor, em $\Omega ; \mathrm{t}=$ tempo em segundos (s); $C p_{c a l}=$ calor específico do calorímetro $\left(\mathrm{cal} / \mathrm{g}^{\circ} \mathrm{C}\right) ; \mathrm{T}_{1}=$ Temperatura inicial $\left({ }^{\circ} \mathrm{C}\right) ; \mathrm{T}_{2}=$ Temperatura final $\left({ }^{\circ} \mathrm{C}\right) ; \mathrm{Cp}$ am $=$ calor específico da amostra $\left(\mathrm{cal} / \mathrm{g}^{\circ} \mathrm{C}\right) ;$

Os registros das temperaturas foram realizados utilizando a plataforma de prototipagem Arduino, utilizando uma linguagem de programação padrão $(\mathrm{C} / \mathrm{C}++)$. Foi utilizado uma placa Arduino Uno, um termômetro eletrônico LM35DZ e um capacitor de leve capacitância que tem a função de filtrar os picos de leituras naturais do termômetro. O termômetro foi adaptado com uma extensão dos fios. Como fonte de energia térmica foi utilizada uma fonte de notebook com uma tensão de 12,3 W, conforme apresentado na fotografia da Figura 2.

Figura 1. Esquema montado para leitura de temperatura.

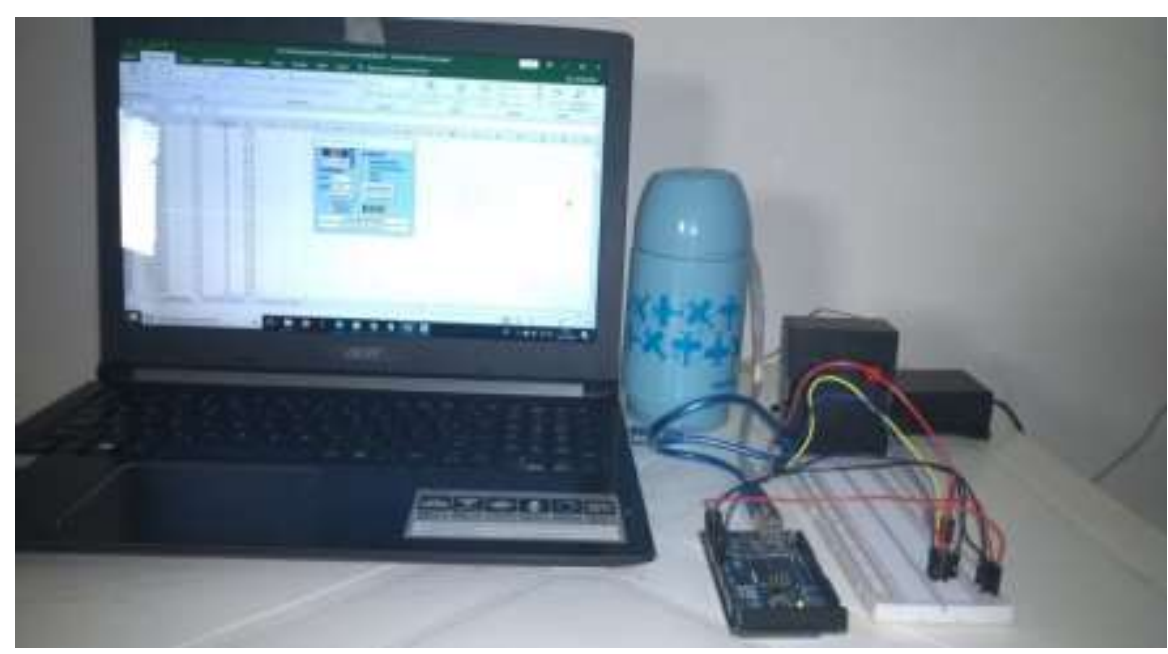

\section{RESULTADOS E DISCUSSÕES}

\subsection{Caracterização da polpa de açaí}

A caracterização do açaí foi realizada considerando os teores de umidade, proteínas, lipídeos, cinzas, $\mathrm{pH}$, sólidos solúveis e densidade, conforme apresentado na Tabela 1. A Instrução Normativa $\mathrm{N}^{\circ} 1$ de 07 de Janeiro de 2000, regulamenta os padrões de identidade e qualidade para polpas de frutas, a quantidade de sólidos solúveis foi o único parâmetro avaliado que está em desconformidade com a mesma. O valor encontrado em ${ }^{\circ}$ Brix $(3,2)$ neste estudo mostra-se muito inferior ao que preconiza a legislação brasileira, entretanto, este valor pode 


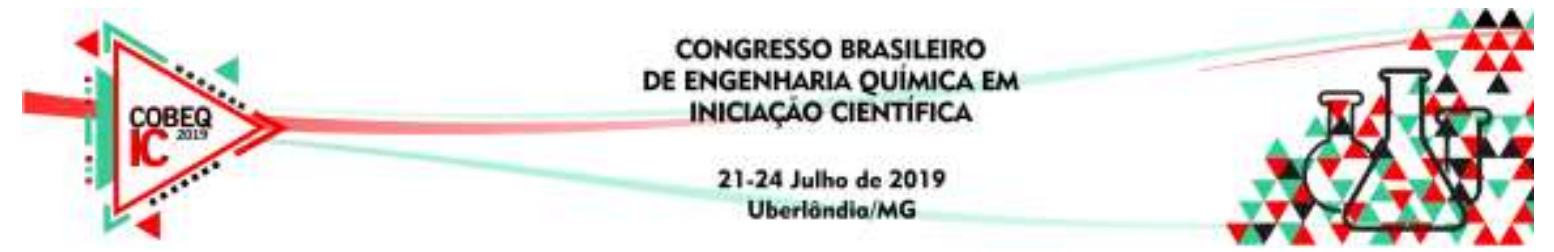

estar relacionado à colheita do fruto, que ocorreu em época de fortes chuvas na região. Pereira et al. (2017), ao avaliarem a polpa de açaí encontraram alguns valores de ${ }^{\circ}$ Brix aproximados e outros menores ao obtido neste trabalho, umidade e $\mathrm{pH}$ similares aos encontrados neste estudo.

Tabela 1 - Composição físico-química dos frutos de açaí

\begin{tabular}{lccc} 
& & \multicolumn{2}{c}{ Instrução Normativa } \\
\cline { 3 - 4 } Composição & Experimental & Mínimo & Máximo \\
\hline Umidade $(\%)$ & $83,97 \pm 0,03$ & - & - \\
Proteína $\left(\mathrm{g} / 100 \mathrm{~g}_{\mathrm{ss}}\right)$ & $7,97 \pm 0,18$ & 5 & - \\
Lipídeos $\left(\mathrm{g} / 100 \mathrm{~g}_{\mathrm{ss}}\right)$ & $24,39 \pm 1,47$ & 20 & 60 \\
Cinzas $\left(\mathrm{g} / 100 \mathrm{~g}_{\mathrm{ss}}\right)$ & $3,24 \pm 0,041$ & - & - \\
Sólidos solúveis $\left({ }^{\circ}\right.$ Brix $)$ & $3,2 \pm 0$ & 40 & 60 \\
$\mathrm{pH}$ & $5,41 \pm 0$ & 4 & 6,2 \\
\hline ss - matéria seca & & &
\end{tabular}

Os valores médios encontrados estão de acordo com os determinados pelo MAPA, sendo similares aos obtidos por Pereira et al. (2017) e superiores aos apresentados por Tonon, Brabet e Hubinger (2010) para polpa de açaí congelada. Isso pode ser explicado pelo fato da composição do açaí variar de acordo com o período da coleta e com o grau de maturação dos frutos. Com o amadurecimento, o teor de lipídeos aumenta, enquanto as cinzas, proteínas e açúcares totais são reduzidos (GORDON et al., 2012).

\subsection{Determinação do calor específico}

Conhecida a capacidade calorifica do calorímetro, foi possível validar o método determinando o calor especifico da água, a quantidade de calor absorvida pela amostra, é a diferença entre a energia total fornecida ao sistema e a absorvida pelo calorímetro. A Figura 2 mostra a quantidade de calor por unidade de massa de água em função da temperatura.

Figura 2. Quantidade de calor por unidade de massa água em função da temperatura.

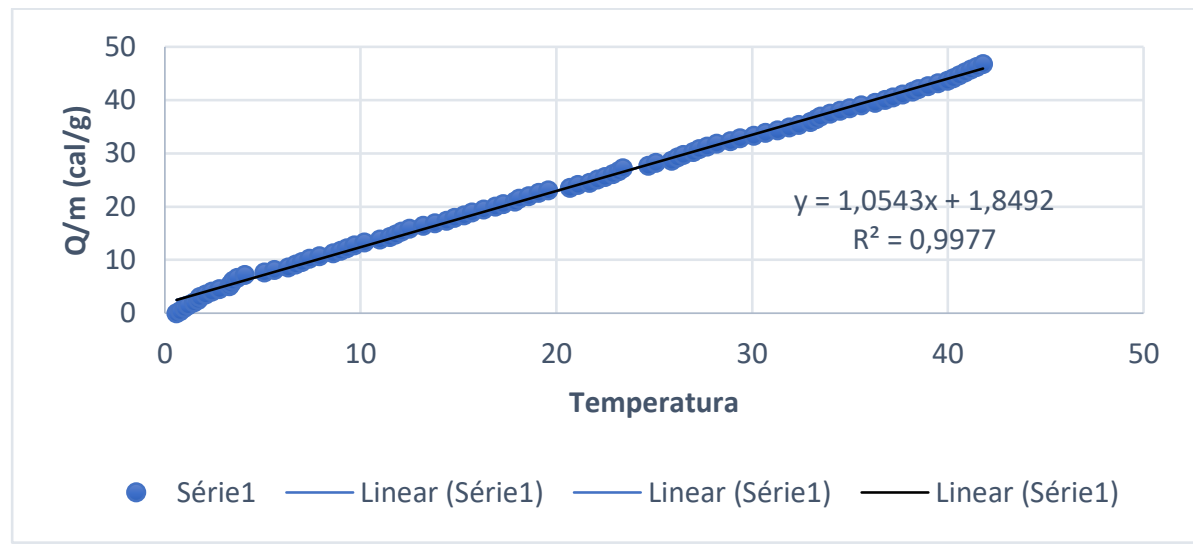

A relação $\mathrm{Q} / \mathrm{m}$ vs. T é descrita por um ajuste linear onde o calor específico da água equivale ao coeficiente angular da reta, $1,054 \mathrm{cal} / \mathrm{g}^{\circ} \mathrm{C}$. $\mathrm{O}$ resultado é muito próximo ao 


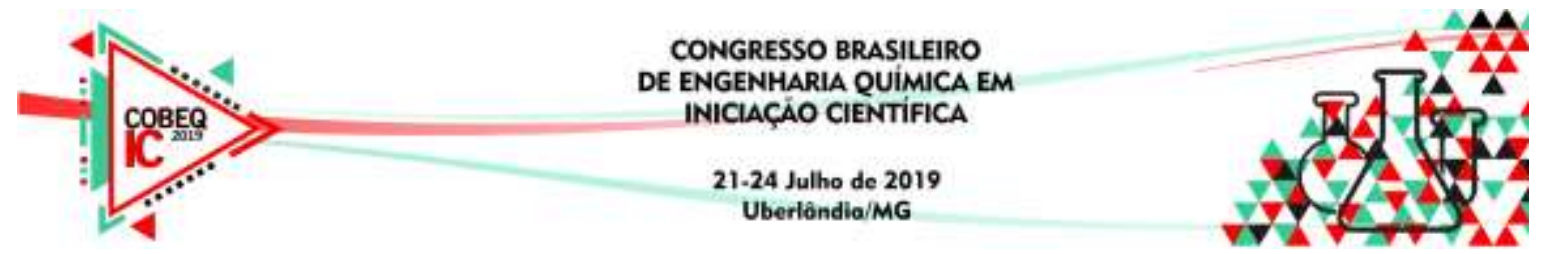

encontrado por vários autores da literatura (Almeida, 2012; Ferraz et al, 2018), que encontraram valores de $1,04 \mathrm{cal} / \mathrm{g}^{\circ} \mathrm{C}$ e $1,058 \mathrm{cal} / \mathrm{g}^{\circ} \mathrm{C}$. Esses resultados indicam a eficiência do método e do sistema desenvolvido.

Para o açaí também foi realizada uma regressão linear de $(\mathrm{Q} / \mathrm{m})$ vs. T, onde o coeficiente angular da reta corresponde ao calor específico da amostra de açaí, conforme informações fornecidas na Figura 3. Os experimentos foram conduzidos na faixa de temperatura de 8 a $45^{\circ} \mathrm{C}$. $\mathrm{O}$ valor obtido para o calor específico da polpa de açaí foi de $0,451 \mathrm{cal} / \mathrm{g}^{\circ} \mathrm{C}$.

Figura 3. Quantidade de calor por unidade de massa em função da temperatura para o açaí.

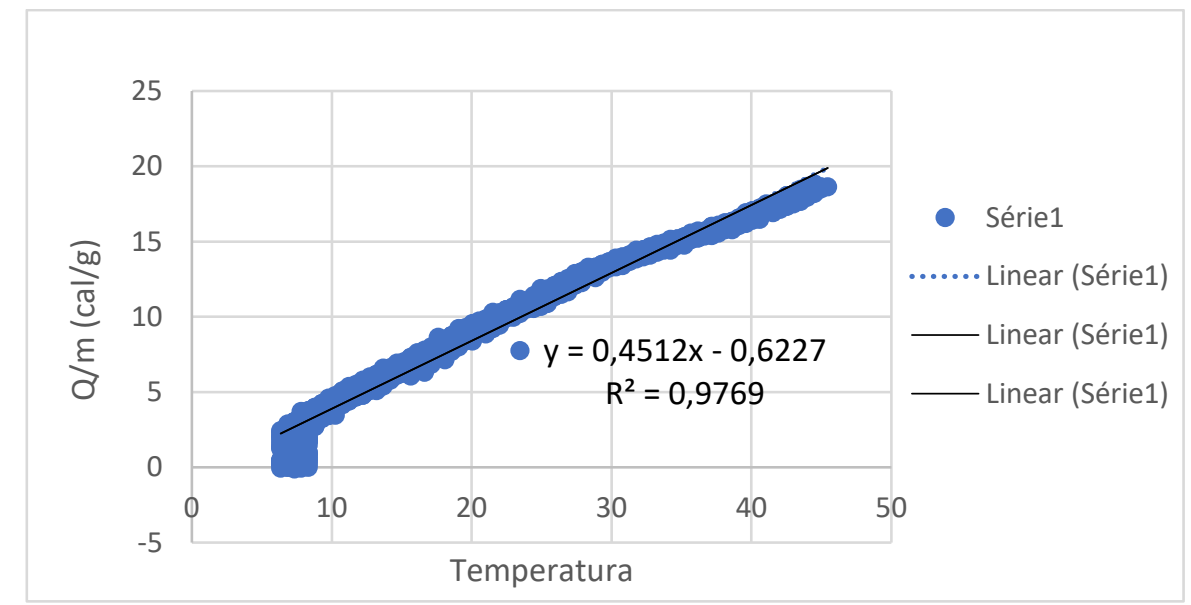

Existem na literatura outros estudos que determinam as propriedades termofísicas de frutas. Pereira (2001) ao determinar o calor específico da polpa de açaí pelo método calorimétrico de misturas encontrou um valor de $0,83 \mathrm{cal} / \mathrm{g}^{\circ} \mathrm{C}$, Ashrae (1981) encontrou para ameixa, cereja, pêra e outras frutas com teor de umidade próxima a do açaí um valor de 0,45 $\mathrm{cal} / \mathrm{g}^{\circ} \mathrm{C}$. Ferraz (2018) ao avaliar polpa de murici (Bysonima crassifólia) in natura utilizando o método descrito neste trabalho encontrou um valor de $\mathrm{Cp}$ de $0,54 \mathrm{cal} / \mathrm{g}^{\circ} \mathrm{C}$. Pode-se inferir que frutas com composição similar podem apresentar propriedades térmicas similares.

\section{CONCLUSÃO}

Os resultados da montagem e determinação da capacidade calorifica do calorímetro foram satisfatórios. Foi possível elaborar e construir um calorímetro simples com uma garrafa térmica e posteriormente determinar sua capacidade calorífica, a qual foi utilizada para os demais cálculos. O valor encontrado para o calor específico da polpa de açaí se aproxima dos valores encontrados para frutas como ameixa, cereja e pêra, frutas que possuem umidade aproximada ao da polpa de açaí.

\section{REFERÊNCIAS}

ALMEIDA, D. S. Determinação experimental do calor específico de matéria mole (soft matter). 2012. 35f. TCC (Graduação) - Curso de Tecnologia em Processos Químicos, Universidade Tecnológica Federal do Paraná, Apucarana, 2012. 


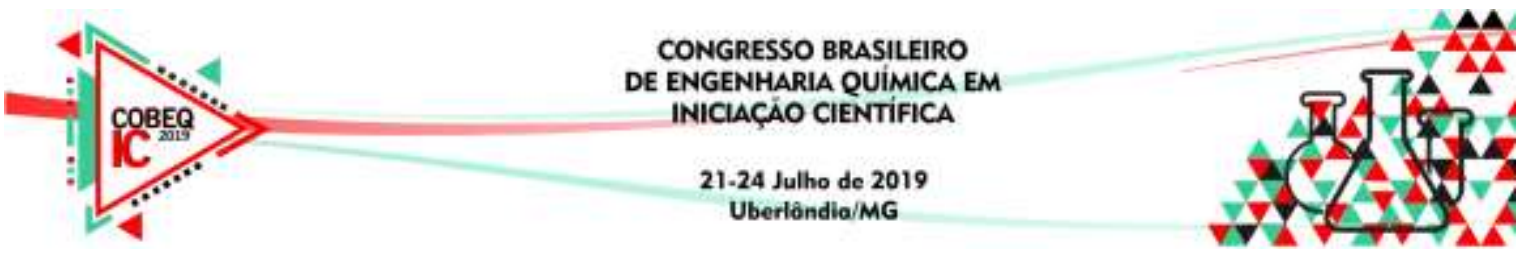

ASHRAE. 1981. ASHRAE Handbook-Fundamentals. Atlanta, Georgia: American Society of Heating, Refrigerating and Air-Conditioning Engineers.

BRASIL. Ministerio da Agricultura, Pecuaria e Abastecimento. Instrução Normativa $N^{\circ} 1$, de 07 de janeiro de 2000. Aprova padrões de identidade e qualidade para polpas de frutas. Diario Oficial [da] Republica Federativa do Brasil, Brasilia, DF, 10 jan. 2000, Seção 1. p.54.

CEDRIM, P. C. A. S.; BARROS, E. M. A.; NASCIMENTO, T. G. do. Propriedades antioxidantes do açaí (Euterpe oleracea) na síndrome metabólica. Brazilian Journal Of Food Technology, Campinas, v. 21, p.1-7, 16 ago. 2018. FapUNIFESP (SciELO). http://dx.doi.org/10.1590/1981-6723.09217.

FERRAZ, F. de S. "Determinação do calor específico do murici (byrsonima crassifólia) a diferentes temperaturas e concentrações de sólidos solúveis”. 2018. 48 f. TCC (Graduação) - Curso de Engenharia de Alimentos, Universidade Federal do Tocantins, Palmas, 2018.

FERRAZ, F. S.; DIAS, A. C. P.; OLIVEIRA, J. C. S. R.; DALLASTRA, E. D. G.; JESUS, M. M. A.; GRAJALES, L. M. ; "Determinação do calor específico do murici (Byrsonima crassifólia) a diferentes temperaturas.", p. 2719-2722 . In:. São Paulo: Blucher,2018. ISSN 2359-1757, DOI 10.5151/cobeq2018-PT.0720

FREITAS, B. et al. Características Físico-químicas, Bromatológicas, Microbiológicas e Microscópicas de Polpas de Açaí (Euterpe oleraceae) Congeladas do Tipo B. Journal Of Applied Pharmaceutical Sciences - Japhac, Governador Valadares, v. 2, n. 2, p.2-13, 12 ago. 2015.

GORDON, André et al. Chemical characterization and evaluation of antioxidant properties of Açaí fruits (Euterpe oleracea Mart.) during ripening. Food Chemistry, vol. 133, p. 256-263, 2012.

LUTZ, I. A. Métodos físico-químico para análise de alimentos. São Paulo: Instituto Adolfo Lutz, 2008.

MOURA, S. C. S. R. et al. Propriedades físicas e reológicas de produtos à base de frutas. Food Technol, Campinas, v. 19, n. 8, p.1-8, nov. 2016.

PEREIRA, E. R. S. et al. Análises físico-químicas da polpa de açaí (Euterpe oleraceae Mart.) comercializada no município de salvaterra e soure. in: Congresso brasileiro de química, 57. Gramado, 2017.

SILVA, F. S. et al. Avaliação higiênico-sanitária dos estabelecimentos com o selo "Açaí Bom" da Vigilância Sanitária. Brazilian Journal Of Food Research, Campo Mourão, v. 4, n. 8, p.157169, dez. 2017.

SOUZA, Paula Gimenez de. Produção e caracterização de açaí (Euterpe oleracea Mart.) desidratado em pó por cast-tape drying. 2015. 143 f. Dissertação (Mestrado) - Curso de Engenharia de Alimentos, Universidade Federal de Santa Catarina, Florianópolis, 2015.

TONON, Renata V.; BRABET, Catherine; HUBINGER, Míriam D. Anthocyanin stability and antioxidant activity of spray-dried açai (Euterpe oleracea Mart.) juice produced with different carrier agents. Food Research International, vol. 43, p. 907-914, 2010. 